\title{
HEALTH INFORMATION-SEEKING BEHAVIOR AMONG HYPOTHYROID PATIENTS AT SAVEETHA MEDICAL COLLEGE AND HOSPITAL
}

\author{
Perumal S.S ${ }^{1,2}$, Prasad $S^{2}$, Surapaneni K.M ${ }^{1,2}$, Joshi $A^{3}$
}

ABSTRACT

BACKGROUND: Hypothyroidism causes considerable morbidity. Low knowledge coupled with inadequate health literacy may lead to poor prevention and management. This study aimed to assess health information-seeking behavior and hypothyroid knowledge among South Indian hypothyroid patients.

METHODS: This cross-sectional study was conducted in October 2013 in Saveetha Medical College, Chennai, India. Hundred clinically diagnosed hypothyroid patients $\geq 18$ years were interviewed in a hospital using a 57-item questionnaire to gather information on their socio-demographics, self-reported disease history, hypothyroid-related knowledge, health information sources, health literacy and health information-seeking behavior. Hypothyroidism was assessed by free T3 and T4 levels.

RESULTS: Mean age of participants was 38 years $(S D=12)$ with median age of 39.5 years, majority of the participants being females (77\%) and living in urban setting (52\%). Mean free T3 level was $0.0137 \mathrm{ng} / \mathrm{dl}(\mathrm{SD}=0.003)$ and mean free $\mathrm{T4}$ was $0.7 \mathrm{ng} / \mathrm{dl}(\mathrm{SD}=0.06)$. Ninety three percent of the participants received initial hypothyroidism education from their physicians at the time of diagnosis. Half of the participants had incorrect hypothyroidism-related knowledge; similar between both genders. Participants with inadequate health literacy had poor knowledge about the hypothyroidism. Hypothyroidism-related health information was sought almost exclusively from health professionals, predominantly regarding treatment, linked to their faith in qualified medical assistance. Economic status primarily determined healthcare-seeking behavior. Marital status, education level, annual household income and health literacy were significantly associated with knowledge.

CONCLUSION: Participants having higher educational qualification, higher annual household income and adequate health literacy had considerable knowledge about hypothyroidism. Developing multifactorial and tailored health education for patients with marginal or inadequate health literacy is needed. Exploring healthcare institutions as a medium for delivery of such education should be explored.

KEYWORDS: Hypothyroidism, Health Information-seeking Behavior, Knowledge, Health Literacy

DOI: http://dx.doi.org/10.4314/ejhs.v25i2.7

\section{INTRODUCTION}

Thyroid disorders are leading causes of morbidity worldwide and remain underreported causes of premature mortality. These impact current societal and future health of the nation in a manner no other non-communicable disease (NCD) does (1). Hypothyroidism, the most common cause of

\footnotetext{
${ }^{1}$ Saveetha Medical College \& Hospital, Saveetha University, Saveetha Nagar, Chennai, Tamil Nadu

${ }^{2}$ Foundation of Healthcare Technologies Society, New Delhi, India

${ }^{3}$ Center for Global Health and Development, College of Public Health, University of Nebraska Medical Center, USA

Corresponding Author: Ashish Joshi, Email: ashish.joshi@unmc.edu
} 
pathological hormone deficiency (2), results due to the insufficient functioning of the thyroid gland to produce thyroid hormone (3).

The prevalence of hypothyroidism, the most common type of thyroid dysfunction (4), in the developed world is $4-5 \%$ (5). Forty two million people in India suffer from thyroid diseases (6). An overall Indian prevalence ranging from 3.9$5.4 \%(7,8)$ in in-land areas and increasing to $11 \%$ in coastal regions like Chennai has been reported. A clinic-based study had estimated that $80 \%$ of those with thyroid dysfunction had hypothyroidism (7).The prevalence of hypothyroidism depends on geographical location (7) and eating habits $(9,10)$ and is 10 times more common in females (6). Five percent of women show overt hypothyroidism or hyperthyroidism (11). Sub-clinical and overt hypothyroidism is significantly associated with metabolic syndrome patients (12).

General awareness about hypothyroidism in India is poor with half of the population having incorrect knowledge, beliefs and practices regarding hypothyroidism (13). Some of the factors may be lack of qualified physicians treating such patients, less time spent by doctors for patient education, lack of awareness and use of available electronic media to harness information, poor knowledge about reliable sources of information (13). Educational needs are much greater in diseased population with poor health literacy(14). Studies have shown the importance of improving patients' knowledge through education and the associated benefits of improving compliance with health care appointments and medications for patients with hypertension and diabetes (14).

An educational booklet intervention on primary hypothyroid patients did not support the routine provision of additional health educational materials in order to improve their adherence or well-being (15).Though educational materials exist for chronic diseases like diabetes and hypertension, there is a lack of widespread evidence on success of hypothyroidism- related educational efforts and its success. Factors potentially affecting the ability of patients to selfmanage hypothyroidism are not very clear. The objective of this study was thus to assess the health information-seeking behavior and knowledge of hypothyroidism among individuals with hypothyroidism at Saveetha Medical College, Chennai, India.

\section{MATERIALS AND METHODS}

This was an exploratory cross-sectional study conducted in Saveetha medical college and hospital, Chennai in Southern state of India in October 2013. Individuals who were aged 18 years and above, attending outpatient department (OPD) of medical college, diagnosed with hypothyroidism and agreeing to give voluntary written informed consent were included in the study. Individuals with mental and physical challenges making it difficult to participate in the study or were involved in other clinical trials were excluded. The study protocol was approved by the Institutional Review Board (IRB) of the Foundation of Healthcare Technologies Society (FHTS), New Delhi (IRB\#FHTS/011/2013).

All patients with history of hypothyroidism or symptoms of hypothyroidism attending the Outpatient Department of the hospital were selected and subjected to biochemical testing of thyroid hormones. Free T3 and T4 levels were assessed and those participants with T3 level less than $0.2 \mathrm{ng} / \mathrm{dl}$ and T4 level less than $0.7 \mathrm{ng} / \mathrm{dl}$ (16) were confirmed with hypothyroidism and deemed eligible to participate in the study. A convenient sample of 100 individuals was thus enrolled.

Data Collection: A 57-items interview schedule was used to collect information on the following variables: socio-demographics (age, gender, family structure, household location, number of household members, annual household income, marital status, education and occupation of participants) (17); hypothyroidism knowledge assessed by a 10 questions tool (13); self-reported disease history(history of hypothyroidism information was also gathered about individuals past history of hypothyroidism education and existing sources of hypothyroidism related information) (18); health literacy assessed by 5 screening questions ((i) How often do you have problems learning about your medical conditions because of difficulty in understanding written information (e.g. books, magazine and written prescription), (ii) How often do you have difficulty understanding information from your healthcare provider (like a doctor, nurse, or health worker) (Oral communication), (iii) How often are 
the directions on medication bottles difficult to understand for you, (iv) How often are you not sure about how to take your medication correctly and (v) How often do you need to have someone help you when you read instructions, pamphlets, or other written material from your doctor or pharmacy) (19) to identify patients with inadequate, adequate or marginal health literacy; health information-seeking behavior assessed by open-ended questions under 6 domains (health seeking behavior, hypothyroidism-related information sources and credibility, use of technology and challenges for seeking information on hypothyroidism, satisfaction with medical care, consistency and reliability of information sources and external factors determining hypothyroidism related management decision) (20).

Statistical Analysis: Quantitative descriptive analysis was performed using univariate statistics to report means and standard deviations for the continuous variables and frequency distribution for the categorical variables. Correlation, t-statistic and 1-way Analysis of Variance were performed to compare differences in the continuous variables. Chi-square and Fisher Exact analyses were performed to compare the frequency of categorical variables. Analysis of hypothyroidism related knowledge was done by scoring every correct response as one and incorrect response as zero. The total was added and then he mean was compared for the independent variables. Analysis of qualitative data was done by extracting common themes from categories after textual data were coded. All analyses were performed using Microsoft Excel 2007 and SPSS version 16.

\section{RESULTS}

Majority of participants were females $(71 \%)$ with an average age of 38 years $(S D=12)$ and median age of 39.5 years. More than half of the participants were married $(71 \%)$, living in nuclear families $(75 \%)$ with an average and median family size of $4(\mathrm{SD}=1)$. There was not demonstrable difference in locational distribution of the participants (urban $=52 \%$; rural $=48 \%$ ). Nineteen percent of the study participants were educated up to high school with $38 \%$ of them having an annual income between 1,00,001-5,00,000 Indian National Rupee (INR) (Table 1).
Disease History: Mean free T3 level was $0.0137 \mathrm{ng} / \mathrm{dl}(\mathrm{SD}=0.003)$ and mean free $\mathrm{T} 4$ was $0.7 \mathrm{ng} / \mathrm{dl} \quad(\mathrm{SD}=0.06)$. Majority $(44 \%)$ were diagnosed with hypothyroidism in the past 1-5 years, $35 \%$ of the participants had hypothyroidism from more than 5years and $21 \%$ participants diagnosed to have hypothyroidism within the recent 1 year. Majority (93\%) of the participants received some form of hypothyroidism education from their doctors at the time of diagnosis; other sources of initial hypothyroidism education were nurse (11\%), dietitian (3\%) and hypothyroidism educator (3\%).

General health-related information sources: Major health-related information sources were friends $(56 \%)$, family (51\%), and healthcare professionals $(36 \%)$. Other sources included radio/television (28\%), newspaper/books/magazine/scientific journal (24\%) and internet/mobile phone (21\%).

All participants managed their hypothyroidism by use of oral medication. Fortyfive percent of participant's defined hypothyroidism correctly; $53 \%$ of the participants identified symptoms of hypothyroidism correctly. Almost half of the participants knew cabbages, cauliflowers and soya should be avoided in case of hypothyroidism. Thirty-four percent of participants believed iodized salt to be a natural treatment for hypothyroidism. Thirty-one percent of the participants had correct knowledge of taking thyroid medication. Seventy-nine percent of the participants had correct knowledge that thyroid stimulating hormone (TSH) test was sufficient for monitoring treatment. A mere $6 \%$ of the participants had a belief in alternative medicine systems like AYUSH (Ayurveda, Yoga, Unani, Siddha, and Homeopathy).

Majority of study participants $(70 \%)$ had problems learning about their medical condition because of difficulty in understanding written information. A little more than half of the participants $(52 \%)$ were always or sometimes unsure about taking medication correctly. Thirty percent of the participants always or often needed help for reading instructions, pamphlets, or other written material from medical professionals (Figure 1). 
Table 1: Socio-demographic characteristics of study participants

\begin{tabular}{|c|c|c|c|}
\hline Variables & Total & Rural $(\mathrm{N}=42)$ & Urban $(N=58)$ \\
\hline Age category & Mean $=38, \mathrm{SD}=12$ & Mean $=36.79, \mathrm{SD}=13.4$ & Mean $=39, \mathrm{SD}=10.9$ \\
\hline $18-27$ years & 21 & $11(52.4 \%)$ & $10(47.6 \%)$ \\
\hline 28-37 years & 23 & $12(52.2 \%)$ & $11(47.8 \%)$ \\
\hline $38-47$ years & 31 & $7(22.6 \%)$ & $24(77.4 \%)$ \\
\hline $48-57$ years & 20 & $10(50.0 \%)$ & $10(50.0 \%)$ \\
\hline $58-67$ years & 5 & $2(40.0 \%)$ & $3(60.0 \%)$ \\
\hline \multicolumn{4}{|l|}{ Gender } \\
\hline Female & 77 & $28(36 \%)$ & $49(64 \%)$ \\
\hline Male & 23 & $14(61 \%)$ & $9(39 \%)$ \\
\hline \multicolumn{4}{|l|}{ Type of family } \\
\hline Joint & 25 & $16(64 \%)$ & $9(36 \%)$ \\
\hline Nuclear & 75 & $26(35 \%)$ & $49(65 \%)$ \\
\hline Total number of household members & Mean $=4.1, \mathrm{SD}=1.6$ & Mean $=4.5, \mathrm{SD}=2.1$ & Mean $=3.9, \mathrm{SD}=1$ \\
\hline$\leq 4$ & 70 & $24(34 \%)$ & $46(66 \%)$ \\
\hline$>4$ & 30 & $18(60 \%)$ & $12(40 \%)$ \\
\hline \multicolumn{4}{|l|}{ Annual income household } \\
\hline$\leq 1,00,000$ & 23 & $22(96 \%)$ & $1(4 \%)$ \\
\hline $1,00,001-5,00,000$ & 38 & $13(34 \%)$ & $25(66 \%)$ \\
\hline $5,00,001-10,00,000$ & 19 & $4(21 \%)$ & $15(79 \%)$ \\
\hline$\geq 10,00,001$ & 20 & $3(15 \%)$ & $17(85 \%)$ \\
\hline \multicolumn{4}{|l|}{ Marital Status } \\
\hline Married & 71 & $24(34 \%)$ & $47(66 \%)$ \\
\hline Single & 17 & $10(59 \%)$ & $7(41 \%)$ \\
\hline Widow/widower & 8 & $5(62.5 \%)$ & $3(37.5 \%)$ \\
\hline Divorced/separated & 4 & $3(75 \%)$ & $1(25 \%)$ \\
\hline \multicolumn{4}{|l|}{ Education status } \\
\hline No schooling/no formal education & 19 & $14(74 \%)$ & $5(26 \%)$ \\
\hline $\begin{array}{l}\text { Primary (1-5), middle (6-8), high } \\
(9-10)\end{array}$ & 18 & $9(50 \%)$ & $9(50 \%)$ \\
\hline $\begin{array}{l}\text { intermediate (11-12), some college, } \\
\text { Post high school diploma but no } \\
\text { college }\end{array}$ & 40 & $16(40 \%)$ & $24(60 \%)$ \\
\hline Graduate or Postgraduate & 23 & $3(13 \%)$ & $20(87 \%)$ \\
\hline \multicolumn{4}{|l|}{ Self-occupation ( $N=99)$} \\
\hline Skilled & 28 & $13(46 \%)$ & $15(54 \%)$ \\
\hline Unemployed & 60 & $17(28 \%)$ & $43(72 \%)$ \\
\hline Unskilled & 11 & $11(100 \%)$ & \\
\hline \multicolumn{4}{|l|}{ Partners occupation $(\mathrm{N}=69)$} \\
\hline Skilled & 45 & $9(20 \%)$ & $36(80 \%)$ \\
\hline Unemployed & 17 & $8(47 \%)$ & $9(53 \%)$ \\
\hline Unskilled & 7 & $7(100 \%)$ & \\
\hline
\end{tabular}




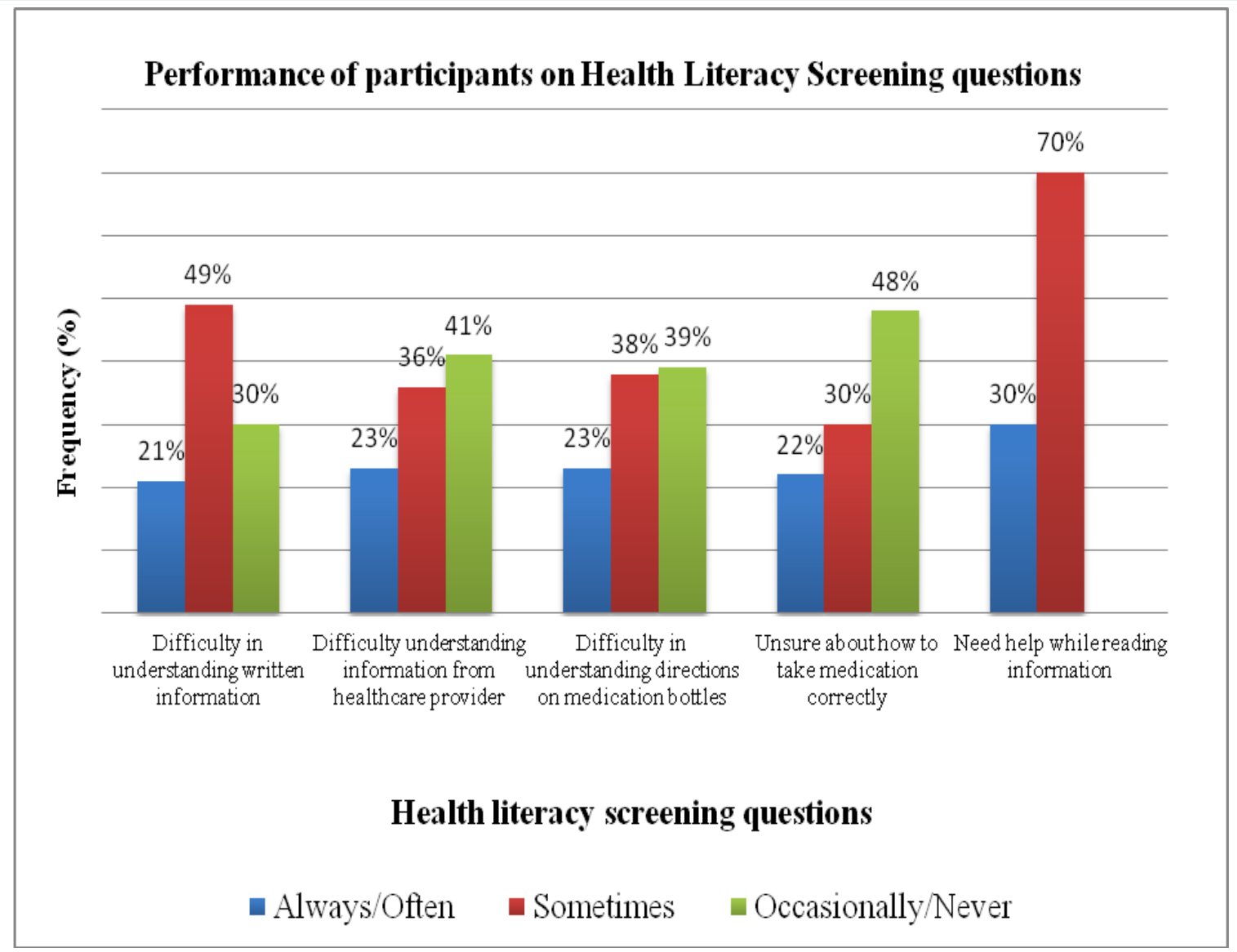

Figure 1: Categorical distribution of study participants on the basis of difficulty in understanding health information at Saveetha Medical College, Chennai, India

Participants in urban settings had higher health literacy and knowledge compared to their rural counter parts. Higher mean knowledge scores were seen among those with adequate health literacy both among rural (mean=5.25; $\mathrm{SD}=2)$ and urban (mean=7.14; $\mathrm{SD}=1$ ) participants (Figure 2).

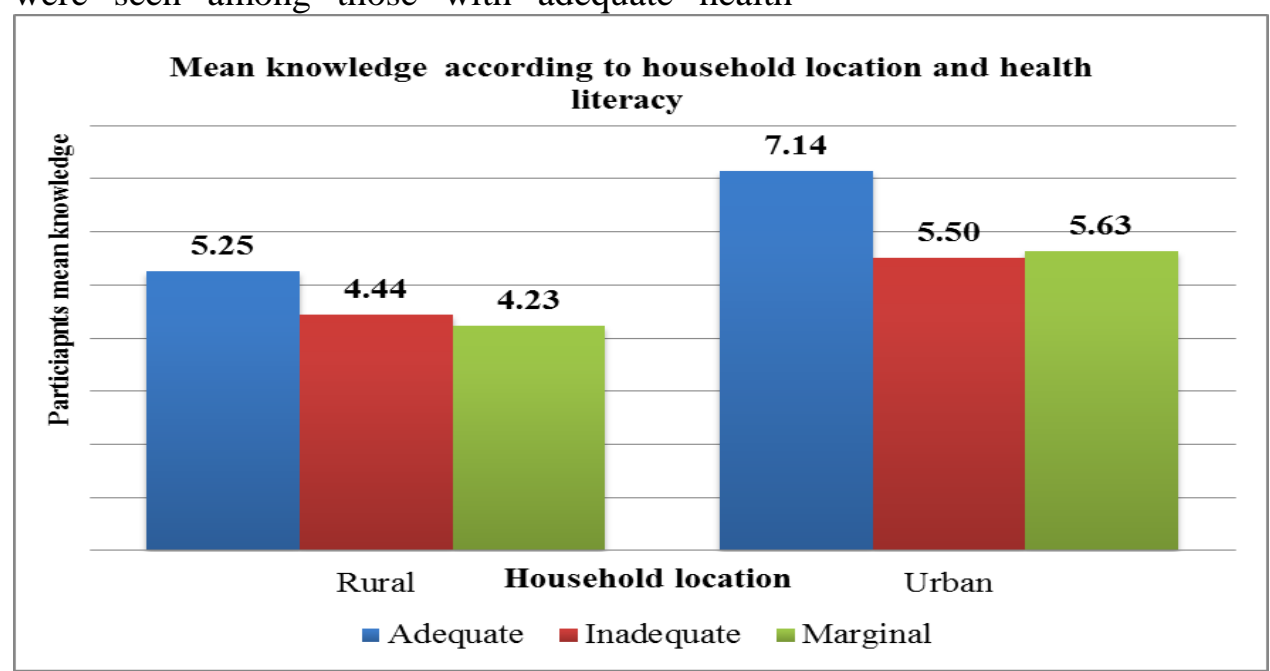

Figure 2: Mean hypothyroidism knowledge score of the study participants interviewed at the OPD of Saveetha Medical College, Chennai, India in accordance to household location and health literacy 
Almost every participant consulted medical professionals or hospital-based sources to seek solutions to their health problem with half of them seeking treatment. Twenty-two percent of the participants had reported that they seek for help because of their belief in medical profession and similar number had reported that they seek help for information and advice. A mere $6 \%$ of the participants also searched help from traditional healers (ayurveda 2\%, homeopathy 4\%). Majority of participants $(74 \%)$ relied on doctors, healthcare professionals to gain information regarding hypothyroidism, only $11 \%$ of the participants used internet and $7 \%$ used books as other source of information (Table 3).

The majority of the participants (93\%) enquired about hypothyroidism only when complications arose. Similarly, majority (91\%) had not read or heard about hypothyroidism. Ninety percent of the participants did not verify if the information received was reliable/credible. Out-of-pocket cost was a relatively major (13\%) external factor impacting the hypothyroidism management.

Highest mean knowledge was found to be among participants in age group 18-27 years (mean $=5.7$; $\mathrm{SD}=1)$ and 38-47 years $($ mean= $5.6, \mathrm{SD}=2)(\mathrm{p}=$ .673).Age, gender, type of family and household settings were not significantly associated with knowledge (Table 4).

Marital Status: Single (mean $=5.9 ; \mathrm{SD}=1.2$ ) participants had the highest knowledge showing a statistically significant association $(\mathrm{p}=$.040) between marital status and knowledge about hypothyroidism.

Education Status: Graduate/ postgraduate participants $($ mean $=6.1 ; \mathrm{SD}=1.7)$ had higher knowledge compared to those with lower or no formal education which was significant $(\mathrm{p}=.013)$.

Annual household income: Participants earning $\geq 10,00,001 \mathrm{INR}$ annually (mean $=6.4 ; \mathrm{SD}=1.2$ ) had higher knowledge compared to lower annual income earnings and was significant $(\mathrm{p}=.000)$.

Health Literacy: Participants with adequate health literacy had higher hypothyroidism knowledge $($ mean $=6.9 ; \mathrm{SD}=1.4)$ than participants with inadequate or marginal health literacy and this was found to be significant $(\mathrm{p}=.000)$.

Multiple regression analysis between knowledge scores and independent variables had shown that age ( $\mathrm{r}=-.014)$ and family size $(\mathrm{r}=-.005)$ were negatively correlated to the hypothyroidism knowledge scores but were not statistically significant. Annual household Income $(\mathrm{p}<.0001)$ and Health literacy scores $(\mathrm{p}<.0001)$ have shown statistical significant correlation with hypothyroidism knowledge scores. Individuals having education level of graduation or above $(\mathrm{p}=.024)$ had shown statistically significant higher knowledge scores. Gender and family type had not shown any significant association with knowledge scores.

\section{DISCUSSION}

This exploratory cross-sectional study was conducted primarily to assess the existing hypothyroidism related knowledge, health seeking behavior and health literacy among hypothyroid patients.

Knowledge about the health problem under study remains an important goal of patient health education programs. Our study had shown that only half of the participants had given correct responses about the hypothyroidism related knowledge questions. This was lower compared to a study by Kannan et al. (13) suggesting that more time and efforts by physicians were needed to be invested to instill a better knowledge and understanding of their disorder. This could be attributed to large number of female respondents having limited options for seeking health information and dependency on family members. Secondly it can also be attributed to level of education as in our study less than one in four had education level of graduation or above. Further, clarifications on misconceptions and counseling about treatment could result in a better compliance and thus outcomes of the patients.

Mean knowledge scores were lowest among participants with inadequate health literacy in both rural and urban participants. A study on diabetic patients (14) similarly reported that many patients with inadequate functional health literacy, even those who have attended formal diabetes education programs, did not know the basics of their disease and self-management skills. Patient education also plays a critical role in facilitating patients' acceptance of their diagnosis and understanding behavioral changes required for active participation in treatment (14). The lowliterate patient cannot fully comprehend medical 
advice using standard patient education methods (14).

Majority of the participants obtained general health-related information from friends and family followed by healthcare professionals and television and radio. However, hypothyroidismspecific information was obtained almost exclusively from physicians and nurses. Earlier studies have reported television as an especially important potential communication medium among elderly diabetic patients (14). A study by Longo et al. (18) on health information-seeking behavior has similarly reported that such patients relied on relationships with friends, family or clinicians to confirm the information they gathered, thus would still prefer physician help and guidance. The Joint Commission on Accreditation of Health Care Organizations (14) mandated that hospitals and other health organizations provide instruction understandable to patients, assess patients' knowledge, and document such educational efforts.

The majority of the participants enquired about hypothyroidism only when medical or other complications arise. Majorities were satisfied with the education they received; however, they did not know if the information they received was reliable/ credible. In the study by Longo et al.(18), patients looked for sources of information which they perceived as credible and made sense to them based on their knowledge and experience with the disease. Satisfaction of the participants with the health education they have received could be attributed to improvements in their disease conditions and their belief in healthcare professional. Limited knowledge about the reliability/credibility of information could be attributed to their limited access to health information sources for comparisons.

Despite the difficulties involved in educating low-literate patients, widespread effort should not be considered hopeless because endeavors to decrease non-communicable disease risk factors among less educated populations have shown some success (14).Communicating medical advice successfully to patients with chronic illnesses who have inadequate or marginal literacy requires providing appropriately written materials, using oral communication, and improving visual presentation (14). Significant effort has focused on improving the quality and appropriateness of printed materials (14). Further research needs to focus on using oral and visual communication to convey necessary medical information through one-on-one teaching, audiotapes, videotapes, and computer multimedia (14).

This study was limited to one hospital with a sample size of 100 which may limit the generalizability of the study findings. Statistical comparisons of gender differentials on hypothyroidism knowledge and information seeking could not be performed due to high volume of female participant in comparison to male participants in the study. Further, a longitudinal study on larger sample sizes may be warranted to establish the causality between knowledge, health literacy and other independent patient factors and health outcomes.

Participants having higher education qualification, higher annual household income and adequate health literacy had considerable knowledge about hypothyroidism. As healthcare professionals were most contacted for information, the potential of harnessing healthcare institutions for delivery of comprehensible education needs to be explored. This needs to be done keeping in mind the challenge of limited time and resources healthcare professionals face. Further research is needed to identify the multiple factors impacting impact knowledge and health seeking behavior of hypothyroid patients. There is an urgent need to review existing national policies and guidelines regarding hypothyroidism-related education. Moreover, the need and usefulness of tailored educational interventions for patients with marginal or inadequate health literacy for possible improvement in their health outcomes should be explored further.

\section{ACKNOWLEDGEMENT}

We would like to express our special thanks to Ms. Jyoti Bala Kasav, M.Sc. (Environmental Science), Environmental Health Researcher at Foundation of Healthcare Technologies Society, New Delhi, India for her general support for the manuscript of the research. 


\section{REFERENCES}

1. Kalra S, Unnikrishnan AG, Baruah MP. Thyroid: Disorders of a lesser gland. Thyroid Res Pract, 2013; 10(2):45.

2. Brown BT, Bonello R, Pollard H, Graham, P. The influence of a biopsychosocial-based treatment approach to primary overt hypothyroidism: a protocol for a pilot study. Trials, 2010; 11(1):106.

3. Hypothyroidism. American Thyroid Association 2012. Retrieved on December 10, 2013, from: http://www.thyroid.org/what-ishypothyroidism/

4. Unnikrishnan AG, Kalra S, Sahay RK, Bantwal G, John M, Tewari N. Prevalence of hypothyroidism in adults: An epidemiological study in eight cities of India. Indian $\mathbf{J}$ Endocrinol Metabol 2013; 17(4):647.

5. Ahmad N, Panthari M, Gupta A, Chandra P, Nafees S. Prevalence of Hypothyroidism among Patients of Meerut, Uttar Pradesh: A Hospital Based Study. Int J Med Sci Public Health, 2013; 2(3):539-542.

6. Choudhury S.D. Hypothyroidism-A common Phenomenon. The Clarion 2012; 1(1).

7. Unnikrishnan AG, Menon UV. Thyroid disorders in India: An epidemiological perspective. Indian $J$ Endocrinol Metabol, 2011; 15(Suppl2): S78.

8. Shah SN, Joshi SR. Think Thyroid. J Assoc Physicians India, 2011; 59:15-20.

9. James, R. Study on the Prevalence of Thyroid Diseases in Ernakulam City and Cherthala Town of Kerala State, India. Int J Sci Res Publ, 2012; 2(3):1-4.

10. Nimmy NJ, Aneesh PM, Narmadha MP, Udupi RH, Binu KM. A Survey on the Prevalence of Thyroid Disorder Induced by Demography and Food Habits in South Indian Population. Indian J Pharm Pract 2012; 5(2): 49-52.

11. John M. Burden of Thyroid Diseases in India. Need for Aggressive Diagnosis, 2008; 13: 334-351.

12. Shantha GP, Kumar AA, Jeyachandran V. et al. Association between primary hypothyroidism and metabolic syndrome and the role of $\mathrm{C}$ reactive protein: a crosssectional study from South India. Thyroid Res, 2009; 2(1):2.
13. Kannan S, Mukundan L, Mahadevan S. et al. Knowledge, Awareness and Practices (KAP) among patients with hypothyroidism attending endocrine clinics of community hospitals in Chennai. Thyroid Res Pract, 2010; 7(1):11.

14. Williams MV, Baker DW, Parker RM, Nurss JR. Relationship of functional health literacy to patients' knowledge of their chronic disease: a study of patients with hypertension and diabetes. Arch Intern Med, 1998; 158(2):166-172.

15. Crilly M, Esmail A. Randomized controlled trial of a hypothyroid educational booklet to improve thyroxine adherence. The $\mathrm{Br} \mathrm{J}$ Gen Pract, 2005; 55(514):362-68.

16. Thyroid function tests. National Endocrine and Metabolic Diseases Information Service ,U.S Department of Health And Human Services, National Institutes of Health, National Institute of Diabetes and Digestive and Kidney Disease,2010. Accessed on December 10, 2013, from: http://www.endocrine.niddk.nih.gov/pubs/thyr oidtests/thyroidtests_508.pdf

17. Sharma A, Gur R, Bhalla P. Kuppuswamy's socioeconomic scale: updating income ranges for the year 2012. Indian J Public Health, 2012; 56(1):103-104.

18. Longo DR, Schubert SL, Wright BA, LeMaster J, Williams CD, Clore JN. Health information seeking, receipt, and use in diabetes self-management. The Ann Fam Med, 2010; 8(4): 334-40.

19. Chew LD, Bradley KA, Boyko EJ. Brief questions to identify patients with inadequate health literacy. Health, 2004;36(8): 588-94.

20. Hjelm K, Atwine F. Health-care seeking behaviour among persons with diabetes in Uganda: an interview study. BMC Int Health Hum Right, 2011;11(1):11. 